\title{
ON PARTITIONS OF PLANE SETS INTO SIMPLE CLOSED CURVES
}

\author{
PAUL BANKSTON ${ }^{1}$
}

\begin{abstract}
We investigate the conjecture that the complement in the euclidean plane $E^{2}$ of a set $F$ of cardinality less than the continuum $c$ can be partitioned into simple closed curves iff $F$ has a single point. The case in which $F$ is finite was settled in [1] where it was used to prove that, among the compact connected two-manifolds, only the torus and the Klein bottle can be so partitioned. Here we prove the conjecture in the case where $F$ either has finitely many isolated points or finitely many cluster points. Also we show there exists a self-dense totally disconnected set $F$ of cardinality c and a partition of $E^{2} \backslash F$ into "rectangular" simple closed curves.
\end{abstract}

Let $X$ and $Y$ be topological spaces. By a topological partition of $Y$ into copies of $X$ we mean a covering of $Y$ by pairwise disjoint sets, each of which is homeomorphic with $X$. In this note $Y$ will be a subset of the euclidean plane $E^{2}$ and $X$ will be the unit circle. A homeomorphic copy of $X$ will be referred to, as usual, as a simple closed curve (scc).

In [1] it is proved that, among the compact connected two-manifolds, only the torus and the Klein bottle can be partitioned into scc's. The key to the proof is the following

1. Theorem (lemma in proof of Theorem 3.3 In [1]). Let $F \subseteq E^{2}$ be finite. Then $E^{2} \backslash F$ can be partitioned into scc's iff $F$ has exactly one point.

Here we are interested in extending Theorem 1; in particular we believe the following is true.

2. Conjecture. Let $F \subseteq E^{2}$ be an infinite set of cardinality less than the continuum $c$. Then $E^{2} \backslash F$ cannot be partitioned into scc's.

In support of this conjecture we have the following result.

3. THEOREM. Let $F \subseteq E^{2}$ be an infinite set of cardinality less then c. If either: (i) $F$ has finitely many isolated points; or (ii) $F$ has finitely many cluster points in $E^{2}$, then $E^{2} \backslash F$ cannot be partitioned into scc's.

In order to prove (3), we will need some auxiliary machinery.

If $S \subseteq E^{2}$ is any scc, let $B(S)$ denote the bounded complementary domain of $S$. By the Jordan Curve Theorem, $S$ is the common boundary of both $B(S)$ and

Received by the editors August 23, 1982 and, in revised form, October 28, 1982.

1980 Mathematics Subject Classification. Primary 54B15, 57N05.

Key words and phrases. Topological partitions, euclidean plane, simple closed curves.

${ }^{1}$ Research partially supported by a Marquette University Summer Faculty Fellowship (1982). 
$E^{2} \backslash(B(S) \cup S)$; so $\overline{B(S)}$, the closure of $B(S)$, is just $B(S) \cup S$. By the Schönflies Theorem, we also have $B(S) \simeq E^{2}$. (This fact will enable us to "relativize" arguments.)

Let $F \subseteq E^{2}$ and assume $\mathcal{S}$ is a partition of $E^{2} \backslash F$ into scc's. We partially order $s$ by $S_{1}<S_{2}$ whenever $\overline{B\left(S_{1}\right)} \subseteq B\left(S_{2}\right)$. Information concerning this ordering is given in the following

4. Lemma. If $F$ is totally disconnected and $\mathbb{M} \subseteq S$ is a maximal chain, then $M=\bigcap\{B(S): S \in \mathcal{O}\}$ is a singleton subset of $F$.

Proof. Note first that since $F$ is totally disconnected, each $S$ encloses points of $E^{2} \backslash F$. Therefore, by maximality, $\mathfrak{T}$ can have no $<$-minimal element; so $M=$ $\cap\{\overline{B(S)}: S \in \mathscr{N}\}$, a chain intersection of continua, is a continuum. Suppose $x \in M \backslash F$. Then $x \in S$ for some $S \in S$; hence $S<S^{\prime}$ for all $S^{\prime} \in$ : $)$, contradicting the maximality of $\mathfrak{N}$. Therefore $M \subseteq F$. Since $F$ is totally disconnected, $M$ must be a singleton.

5. Lemma. Let $1<|F|<c$. Then $S$ is not a chain.

Proof. Suppose $S$ is a chain. We first show that every $x \in F$ is enclosed by a member of $\delta$. Since $E^{2}$ is not compact, $\S$ has no $<$-maximal element; so every $z \in E^{2} \backslash F$ is enclosed by some member of $s$. Let $U=\cup\{B(S): S \in \Omega\}$. Then $E^{2} \backslash F \subseteq U$. If $F_{1}=\{x \in F: x \in B(S)$ for some $S \in \S\}$, then $U=E^{2} \backslash\left(F \backslash F_{1}\right)$. Now $U$ is simply connected, but removal of a nonempty subset of the plane of cardinality $<c$ leaves a multiply connected set. Thus $F_{1}=F$.

Now by Lemma 4, since $F$ is totally disconnected, $\cap\{B(S): S \in \Omega\}$ is a singleton subset of $F$, say $\{y\}$. Let $x \in F \backslash\{y\}, \varsigma_{1}=\{S \in \Omega: x \notin B(S)\}$, and $\varsigma_{2}=\varsigma_{s_{1}}$. We know $\delta_{1} \neq \varnothing$ since $y$ is the only point in every $\overline{B(S)}$. We know $\varsigma_{2} \neq \varnothing$ since $F_{1}=F$. We show $\delta_{2}$ has no $<$-minimal element. For assume otherwise and let $S_{2} \in \mathcal{S}_{2}$ be $<$-minimal. Then $\mathcal{S}_{1}$ is a partition of $B\left(S_{2}\right)\left(\simeq E^{2}\right)$ into scc's. By relativization of the argument showing $F_{1}=F$, we then conclude $x \in B(S)$ for some $S \in \delta_{1}$, a contradiction. Next we show $\delta_{1}$ has no $<$-maximal element. Assuming otherwise, let $S_{1} \in \mathcal{S}_{1}$ be $<$-maximal and set $C=\bigcap\left\{\overline{B(S)}: S \in \mathcal{S}_{2}\right\}$. Then $C$ is a continuum containing $\overline{B\left(S_{1}\right)} \cup\{x\}$. By Urysohn's Lemma there is a continuous map $f: C \rightarrow[0,1]$ (the closed unit interval) taking $\overline{B\left(S_{1}\right)}$ to 0 and $x$ to 1 . Since $C$ is connected, $f$ is surjective and must take $\left.C \backslash \overline{\left(B\left(S_{1}\right)\right.} \cup\{x\}\right)$ onto $(0,1)$. Since $|F|<c$, there must be a point $\left.z \in C \backslash \overline{\left(B\left(S_{1}\right)\right.} \cup F\right)$. Let $z \in S$ for some $S \in \mathcal{S}$. Since $\mathcal{S}_{2}$ has no $<$-minimal element, we know $S<S^{\prime}$ for each $S^{\prime} \in \mathcal{S}_{2}$. Thus $S \in \mathcal{S}_{1}$, so $S \leqslant S_{1}$ by the $<$-maximality of $S_{1}$. This implies $z \in \overline{B\left(S_{1}\right)}$, an impossibility.

Let $U_{1}=\cup\left\{B(S): S \in \delta_{1}\right\}$. Since $\delta_{1}$ has no $<$-maximal element, $U_{1}=$ $\cup\left\{\overline{B(S)}: S \in \mathcal{S}_{1}\right\}$, so $\cup \mathcal{S}_{1} \subseteq U_{1}$. Let $U_{2}=\cup\left\{E^{2} \backslash \overline{B(S)}: S \in \mathcal{S}_{2}\right\}$. Since $\mathcal{S}_{2}$ has no $<$-minimal element, $U_{2}=\cup\left\{E^{2} \backslash B(S): S \in \varsigma_{2}\right\}$, so $\cup \delta_{2} \subseteq U_{2}$. Clearly $U_{1} \cap$ $U_{2}=\varnothing$, so $\left\{U_{1}, U_{2}\right\}$ forms a disconnection of $E^{2} \backslash F$. But sets of cardinality $<c$ cannot separate $E^{2}$. This proves the lemma.

We are now in a position to prove part of Theorem 3.

6. Proposition. Let $F \subseteq E^{2}$ be an infinite set of cardinality less than c. If $F$ has at most one isolated point, then $E^{2} \backslash F$ cannot be partitioned into scc's. 
Proof. Suppose $F$ has no isolated points ( $F$ is "self-dense"), and let $\delta$ be a partition of $E^{2} \backslash F$ into scc's. By Lemma $5 \delta$ is not a chain, so one can find external $S_{1}, S_{2} \in \mathcal{S}$ (i.e. $\left.B\left(S_{1}\right) \cap B\left(S_{2}\right)=\varnothing\right)$ ). By Theorem $1, B\left(S_{1}\right)$ and $B\left(S_{2}\right)$ must intersect $F$ in a self-dense set, so we can relativize the above procedure to $B\left(S_{1}\right), B\left(S_{2}\right)$. This forms the basis for a binary tree construction; hence $\delta$ must have $c$ distinct maximal chains. By Lemma 4, this forces $|F|=c$ and a contradiction.

Suppose $F$ has one isolated point $x$. If $S \in \mathcal{S}$ then $x \in B(S)$ by the above argument. Thus $\S$ is a chain, contradicting Lemma 5 .

In order to prove the rest of Theorem 3, we will need to know more about the ordering $<$. In what follows it will be convenient to view the two-sphere $S^{2}$ as $E^{2} \cup\{p\}$, the plane with a point at infinity. This ploy will enable us to "exchange" old points for the new point $p$.

As usual, let $F \subseteq E^{2}$ and let $\varsigma$ be a partition of $E^{2} \backslash F$ into scc's.

7. Lemma. Suppose $|F|<c$. The following are equivalent.

(i) $\mathcal{S}$ is upwardly directed (i.e. given $S_{1}, S_{2} \in \mathcal{S}$ there is some $S \in \mathcal{S}$ with $S_{1}<S$, $\left.S_{2}<S\right)$.

(ii) No maximal chain in $\delta$ has a $<$-maximal element.

(iii) $\cup\{B(S): S \in \delta\}=E^{2}$.

Proof. Assume (i). Then (ii) follows immediately. Assume (ii). If $x \in E^{2} \backslash F$ then $x \in S$ for some $S \in \mathcal{S}$, so let $S<S^{\prime}$ for some $S^{\prime} \in \mathcal{S}$. Then $x \in B\left(S^{\prime}\right)$, hence $x \in U=\cup\{B(S): S \in \mathcal{S}\}$. Now $U$ is a union of pairwise disjoint connected simply connected open sets and, hence, is simply connected. Thus we may argue as in the proof of Lemma 5, where we showed $F_{1}=F$. Thus (iii) holds. Assume (iii). If $S_{1}$, $S_{2} \in \mathcal{S}$ are given, suppose first $S_{1} \leqslant S_{2}$. Pick $x \in S_{2}$. Then $x \in B(S)$ for some $S \in \mathcal{S}$, hence $S_{2}<S$. On the other hand suppose $S_{1}, S_{2}$ are external and let $\mathfrak{K}_{1}, \mathfrak{K}_{2}$ be maximal chains containing $S_{1}, S_{2}$, respectively. If $\Re_{1} \cap \mathfrak{K}_{2}=\varnothing$, then

$$
\cup\left\{B(S): S \in \Re_{1}\right\} \cap \cup\left\{B(S): S \in \Re_{2}\right\}=\varnothing .
$$

By (iii) this gives a disconnection of $E^{2}$. So let $S \in \Re_{1} \cap \mathfrak{T}_{2}$. Since $\mathfrak{K}_{1}, \mathfrak{R}_{2}$ are chains and $S_{1}, S_{2}$ are external, we have $S_{1}<S, S_{2}<S$ and (i) holds.

We will have use for the following classical results.

8. Lemma. (i) (Mullikin $[4,5]$ ). Let $C_{0}, C_{1}, \ldots$, be a countable collection of pairwise disjoint closed nonseparating subsets of $E^{2}$ (i.e. $E^{2} \backslash C_{n}$ is connected, $n=0,1, \ldots$ ). Then $\cup_{n=0}^{\infty} C_{n}$ is also nonseparating.

(ii) (Sierpiński [3, p. 173]). Let $Y$ be a locally compact connected Hausdorff space. Then $Y$ cannot be partitioned into countably many proper compact subsets. (This result is proved in [3] for $Y$ compact, but is clearly true in the more general situation since locally compact spaces are open in their compactifications.)

(iii) [6, p. 143]. Every connected simply connected open subset of the two-sphere has connected boundary.

9. Lemma. Let $F \subseteq E^{2}$ and let $\delta$ be a partition of $E^{2} \backslash F$ into scc's. If either (i) $F$ is countable, or (ii) $|F|<c$ and $\delta$ has finitely many maximal chains, then $\delta$ is upwardly directed. 
Proof. If $F$ is finite, then $|F|=1$ by Theorem 1 , and $\delta$ is a chain without endpoints. So assume $F$ is infinite. For each $x \in F$ let $\varsigma_{x}=\{S \in \S: x \in B(S)\}$. By Theorem 1 each $S \in \mathcal{S}$ encloses some member of $F$, so $\mathcal{S}=\cup_{x \in F} \mathcal{S}_{x}$, and each $\varsigma_{x}$ is an upwardly closed chain (i.e. $S \in \mathcal{S}_{x}$ and $S<S^{\prime}$ imply $S^{\prime} \in \mathcal{S}_{x}$ ). For each $x \in F$ let $\left.G_{x}=\{x\} \cup \cup \overline{B(S)}: S \in \mathcal{S}_{x}\right\}$. Then $\cup_{x \in F} G_{x}=E^{2}$; and, for all $x, y \in F$ either $G_{x}=G_{y}$ or $G_{x} \cap G_{y}=\varnothing$. We partition $F$ into three subsets: $F_{e}=\left\{x \in F: \varsigma_{x}=\varnothing\right\}$; $F_{m}=\left\{x \in F: \S_{x}\right.$ has a $<$-maximal element $\}$; and $F_{u}=\left\{x \in F: \S_{x} \neq \varnothing\right.$ and has no $<$-maximal element $\}$. Then for each $x \in F, G_{x}$ is a singleton, a closed disk, or a chain union of open disks, respectively, as $x$ is in $F_{e}, F_{m}$, or $F_{u}$.

Suppose $F$ is countable. If there are at least two sets $G_{x}$ for which $x \in F_{u}$, then the plane minus a countable number of pairwise disjoint nonseparating closed sets (either closed disks or singletons) is disconnected, contradicting Lemma 8(i). If $F_{u}=\varnothing$, we contradict Lemma 8(ii). Thus there is exactly one such $G_{x}$, call it $U$. We show $U=E^{2}$, i.e. $F_{e} \cup F_{m}=\varnothing$. Suppose otherwise, and there are only finitely many sets $G_{x}$ for which $x \in F_{m}$. Then $U$ is homeomorphic to $E^{2}$ minus a nonempty countable set, hence multiply connected. Thus there are infinitely many such sets $G_{x}$, and we can enumerate them $D_{0}, D_{1}, \ldots$, so $E^{2}=U \cup F_{e} \cup \cup_{n=0}^{\infty} D_{n}$. Let $B=$ $\operatorname{Bd}(U)=\bar{U} \backslash U$ (the boundary of $U$ ), and let $S_{n}=\operatorname{Bd}\left(D_{n}\right), n=0,1, \ldots$ Any point not in $F_{e} \cup \cup_{n=0}^{\infty} S_{n}$ is either in $U$ or in some $D_{n} \backslash S_{n}$, so $B \subseteq F_{e} \cup \cup_{n=0}^{\infty} S_{n}$. On the other hand, if $x \in S_{n}, V$ is an open set containing $x$, and $D \subseteq V$ is a closed disk containing $x$ in its interior, then $D \nsubseteq D_{m}, m=0,1, \ldots$; hence by Lemma 8(ii), $D$ cannot be covered by $F_{e} \cup \cup_{m=0}^{\infty}\left(D \cap D_{m}\right)$. Thus $D \cap U \neq \varnothing$, so $x \in B$. Similarly if $x \in F_{e}$ then $x \in B$, so the boundary of $U$ is made up of points of $F_{e}$ and the scc's $S_{n}$.

We now view $E^{2}$ as $S^{2} \backslash\{p\}$. In $S^{2}, U$ is still a connected simply connected open set; and its boundary $B^{\prime}$ in $S^{2}$ is either $B$ or $B \cup\{p\}$, depending on whether $U$ is bounded in $E^{2}$. In either case $B^{\prime}$ is a continuum by Lemma 8(iii), and we contradict Lemma 8(ii). Thus $F_{e} \cup F_{m}=\varnothing$ and we can apply Lemma 7 .

Now suppose $|F|<c$ and $\delta$ has finitely many maximal chains. Then there are only finitely many sets $G_{x}$ for which $x \in F_{m}$. Letting $D_{0}, \ldots, D_{m}$ denote these $G_{x}$ 's, and $W=\cup_{x \in F_{u}} G_{x}$, we have $E^{2}=W \cup F_{e} \cup \cup_{n=0}^{m} D_{n}$. Then $W$ is homeomorphic to $E^{2}$ minus a nonempty set of cardinality $<c$, hence multiply connected. This is impossible since the components of $W$ are chain unions of open disks. Thus $F_{m} \cup F_{u}=\varnothing$ and the lemma is proved.

Another step in the proof of Theorem 3 is the following.

10. Proposition. Let $F \subseteq E^{2}$ be an infinite set of cardinality $<$ c. If $F$ has finitely many isolated points then $E^{2} \backslash F$ cannot be partitioned into scc's.

Proof. Let $n$ be the number of isolated points of $F$. By Proposition 6 we can assume $n>1$ and proceed by induction. We write $F=F_{0} \cup\left\{x_{1}, \ldots, x_{n}\right\}$ where the $x_{i}$ 's are isolated, $F_{0}$ is self-dense, and let $S$ be a partition of $E^{2} \backslash F$ into scc's. If $S \in \mathcal{S}$ then Proposition 6 tells us that $B(S) \cap\left\{x_{1}, \ldots, x_{n}\right\} \neq \varnothing$, hence $\mathcal{S}$ has at most $n$ maximal chains. By Lemma 9 we know $U=\bigcup\{B(S): S \in \mathcal{S}\}=E^{2}$.

If $F$ is unbounded in $E^{2}$, we can "exchange $x_{1}$ for $p$ ". Then $\delta$ is a partition of $\left(S^{2} \backslash\left\{x_{1}\right\}\right) \backslash\left(\{p\} \cup\left(F \backslash\left\{x_{1}\right\}\right)\right)$ into scc's. Thus we have added a cluster point and 
lost an isolated point. By our induction hypothesis, this cannot happen; hence $F$ must be bounded. For each $A \subseteq\left\{x_{1}, \ldots, x_{n}\right\}$ let $\varsigma_{A}=\left\{S \in \varsigma: B(S) \cap\left\{x_{1}, \ldots, x_{n}\right\}\right.$ $=A\}, s_{i}=s_{A}$ for $A=\left\{x_{i}\right\}$, and $s^{\prime}=S_{A}$ for $A=\left\{x_{1}, \ldots, x_{n}\right\}$. By the induction hypothesis, $\delta_{A}=\varnothing$ whenever $1 \neq|A|<n$. Thus $s=\varsigma^{\prime} \cup \cup_{i=1}^{n} \varsigma_{i}$. Since $U=E^{2}$ and $F$ is bounded, an easy compactness argument tells us that $F \subseteq S$ for some $S \in \Omega$, hence $S^{\prime} \neq \varnothing$. Now suppose $S \in \Omega$ is such that $B(S) \cap F_{0} \neq \varnothing$, but $F_{1}=F_{0} \backslash B(S) \neq \varnothing$ also. $F_{1}$ has no isolated points and $F_{1} \cup\{p\}$ has at most one isolated point. Exchange $\overline{B(S)}$ for $p . S^{2} \backslash \overline{B(S)} \simeq E^{2}$, so what we get is a partition of $E^{2}$ minus a set of cardinality $<c$ with at most one isolated point into scc's (since $\left.S \in S^{\prime}\right)$. This contradicts Proposition 6.

We thus have the fact that $S \in S^{\prime}$ iff $B(S) \cap F_{0} \neq \varnothing$ iff $F \subseteq B(S)$. Now suppose $S \in \varsigma^{\prime}$. Then $\varsigma_{S}=\left\{S^{\prime} \in \mathcal{S}: S^{\prime}<S\right\}$ is a partition of $B(S) \backslash F$ into scc's; so by relativization there is some $S^{\prime}<S$ enclosing a point of $F_{0}$. Thus $S^{\prime} \in S^{\prime}$ as well, so $s^{\prime}$ has no $<-$-minimal element. Let $C=\bigcap\left\{B(S): S \in \mathcal{S}^{\prime}\right\}=\bigcap\left\{\overline{B(S)}: S \in \mathcal{S}^{\prime}\right\}$. Then. $C$ is a continuum containing $\bar{F}=\bar{F}_{0} \cup\left\{x_{1}, \ldots, x_{n}\right\}$. Since $\bar{F}_{0}$ is a perfect set, we have $\left|\bar{F}_{0}\right|=c$. Thus there is a point $x \in \operatorname{Bd}\left(F_{0}\right)$. Let $S_{x} \in \mathcal{S}$ contain $x$. Then $S_{x} \subseteq C$, so $S_{x} \notin \mathcal{S}^{\prime}$. Thus $S_{x} \in S_{i}$ for some $1 \leqslant i \leqslant n$. Now exchange $\overline{B\left(S_{x}\right)}$ for $p$. Then $\left\{S \in \mathcal{S}: S \$ S_{x}\right\}$ is a partition of $\left(E^{2} \backslash \overline{B\left(S_{x}\right)}\right) \backslash\left(F \backslash\left\{x_{i}\right\}\right)$, and is therefore a partition of $\left(S^{2} \backslash \overline{B\left(S_{x}\right)}\right) \backslash\left(\{p\} \cup\left(F \backslash\left\{x_{i}\right\}\right)\right)$. Now $\{p\} \cup\left(F \backslash\left\{x_{i}\right\}\right)$ has $n$ isolated points but is unbounded (since $x \in \operatorname{Bd}\left(F_{0}\right)$ ). This is impossible by earlier remarks, and the proof is complete.

To finish the proof of Theorem 3 we have

11. Proposition. Suppose $F \subseteq E^{2}$ is an infinite set with finitely many cluster points in $E^{2}$. Then $E^{2} \backslash F$ cannot be partitioned into scc's.

Proof. Let $n$ be the number of cluster points of $F$. Then $F$ is scattered and (since $E^{2}$ is hereditarily Lindelöf), therefore, countable. Let $\varsigma$ be a partition of $E^{2} \backslash F$ into scc's. We induct on $n$. If $n=0$ then $F$ is closed discrete, so no $S \in \mathcal{S}$ can enclose infinitely many points of $F$. By Lemma 9 some member of $\delta$ must enclose exactly $k$ points of $F$ for some $k>1$, contradicting Theorem 1 .

So assume $n>0$ and $x$ is a cluster point of $F$. By Lemma 9 there is some $S_{x} \in \mathcal{S}$ with $x \in B\left(S_{x}\right)$. First suppose $x \in F$ and define $F_{x}=B\left(S_{x}\right) \cap F$. Then $F_{x}$ is infinite and bounded. Let $\mathcal{S}^{\prime}$ be a partition of $E^{2} \backslash\left(\overline{B\left(S_{x}\right)}\right)$ into scc's, together with $\{S \in \mathcal{S}$ : $\left.S \leqslant S_{x}\right\}$. Then $\mathcal{S}^{\prime}$ is a partition of $E^{2} \backslash F_{x}$. Exchanging $x$ for $p$, we get that $\mathcal{S}^{\prime}$ is also a partition of $\left(S^{2} \backslash\{x\}\right) \backslash\left(\{p\} \cup\left(F_{x} \backslash\{x\}\right)\right)$. Since the number of cluster points has been reduced by one, we violate our inductive hypothesis. So it must be the case that $x \notin F$, hence there is some $S_{0} \in \mathcal{S}$ with $x \in S_{0}$. Then $S_{0}<S_{x}$. Now define $\mathcal{S}^{\prime}$ to be a partition of $E^{2} \backslash \overline{B\left(S_{x}\right)}$ into scc's, together with $\left\{S \in \mathcal{S}: S \leqslant S_{x}, S \$ S_{0}\right\}$, and let $F_{x}=\left(B\left(S_{x}\right) \cap F\right) \backslash B\left(S_{0}\right)$. By the inductive hypothesis relativized to $B\left(S_{0}\right), S_{0}$ can enclose only one point of $F$. Thus $F_{x}$ is again infinite and bounded. Then $\mathcal{S}^{\prime}$ is a partition of $\left(E^{2} \backslash \overline{B\left(S_{0}\right)}\right) \backslash F_{x}$. Now exchange $\overline{B\left(S_{0}\right)}$ for $p$. Then $\mathcal{S}^{\prime}$ is also a partition of $\left(S^{2} \backslash \overline{B\left(S_{0}\right)}\right) \backslash\left(\{p\} \cup F_{x}\right)$. Again we have exchanged a cluster point for an isolated point, so the inductive hypothesis is violated. This completes the proof of the proposition and the proof of Theorem 3. 
The hypothesis " $|F|<c$ " in Theorem 3 is crucial. Lest the reader be tempted into conjecturing that the weaker hypothesis " $F$ is totally disconnected" will do, we offer the following construction, due to R. Fox [2].

12. TheOREM (R. Fox). There exists a self-dense totally disconnected set $F$ of cardinality $c$ and a partition of $E^{2} \backslash F$ into "rectangular" scc's.

Proof. The easiest way to see this is to construct a family of pairwise disjoint (rectangularly shaped, for convenience) scc's in $E^{2}$, let $F$ be the set of points not covered, and observe that $F$ has the stated properties. The process uses a double induction on the integers, and we describe the essential steps. All scc's in the construction may be taken to be the boundaries of rectangles with sides parallel to the coordinate axes.

Step 1. Partition $E^{2} \backslash([0,3] \times[0,1])$ into scc's.

Step 2. Throw in the new scc's $(\{0,1\} \times[0,1]) \cup([0,1] \times\{0,1\})$ and $(\{2,3\} \times$ $[0,1]) \cup([2,3] \times\{0,1\})$, thus creating three "holes" to fill. The outer ones, called " $G$-holes", are copies of $(0,1)^{2}$; and the inner one, called an " $H$-hole", is a copy of $(0,1) \times[0,1]$.

Step 3 (partially filling $G$-holes). This creates two new $G$-holes and an $H$-hole, all of smaller diameter. For simplicity, let $G=(0,5) \times(0,3)$ be a typical $G$-hole. Then fill in

$$
G \backslash(((1,2) \times(1,2)) \cup((2,3) \times[1,2]) \cup((3,4) \times(1,2)))
$$

as in Steps 1 and 2.

Step 4 ( partially filling $H$-holes). This step creates three new $G$-holes and four new $H$-holes, all of smaller diameter. For simplicity, let $H=(0,9) \times[0,5]$ be a typical $H$-hole. First we fill in the closed annulus $([3,6] \times[0,5]) \backslash((4,5) \times(2,3))$, creating a smaller $G$-hole. The two outer $H$-holes are narrower, but not of diminished height (we want $F$ to be totally disconnected). By filling in the annuli

$$
((0,3) \times(1,4)) \backslash((1,2) \times(2,3)) \text { and }((6,9) \times(1,4)) \backslash((7,8) \times(2,3)),
$$

we get two new $G$-holes plus four new $H$-holes in the corners.

Steps 1-4 outline the induction; and a tree $\mathcal{T}$, whose nodes are $G$-holes and $H$-holes, is thus produced. Let $F$ be the set of points contained in the intersections of branches of $\mathcal{T}$. Not every branch has nonempty intersection, so $F$ is not a Cantor set. However it is clear that $F$ is dense in a Cantor set.

13. REMARKS. (i) In the proof of Theorem 12 the partition $\delta$ of $E^{2} \backslash F$ has cardinality $c$. It would be interesting to know for which $F$ this is necessarily the case.

(ii) One could also conjecture the analogue of Conjecture 2 for $F$ compact totally disconnected. For example an affirmative answer even in the case $F$ is a Cantor set would show that Theorem 12 is pretty sharp. What we know at this point is very sketchy. (For example, if $\mathcal{S}$ is a partition of $E^{2} \backslash F$ into scc's an argument similar to the proof of Lemma 9 shows that $F \subseteq B(S)$ for some $S \in \mathcal{S}$, so $\mathcal{S}$ is upwardly directed.) Also the referee has observed that if we can prove the conjecture for $F$ compact totally disconnected, then by a theorem of R. L. Moore [3, p. 533] on upper semicontinuous decompositions of $E^{2}$ and $S^{2}$ into compact nonseparating subsets, 
we can extend an affirmative answer to cover the case where $F$ is compact with nonseparating components.

(iii) I would like to thank several individuals who have shown interest in this problem area and have shared their ideas. At the risk of giving an embarrassingly incomplete list, I thank especially: Ralph Fox, Fred Galvin, Ronnie Levy, Richard McGovern, Daniel Moran, Neelima Shrikhande, Saul Stahl and the referee.

ADDED IN PROOF. We have recently been able to show that $E^{2} \backslash F$ cannot be partitioned into scc's when $F$ is compact totally disconnected and of cardinality $\neq 1$. Also we can show that if $F$ is any nondense subset of $E^{2}$ and $\mathcal{S}$ is a partition of $E^{2} \backslash F$ into scc's, then $|\delta|=c$. These results will appear in a sequel in this journal.

\section{REFERENCES}

1. P. Bankston and R. McGovern, Topological partitions, General Topology and Appl. 10 (1979), 215-229. MR 80i: 54007.

2. R. Fox (private communication).

3. K. Kuratowski, Topologv, Vol. II, Academic Press, New York, 1966.

4. S. Mazurkiewicz, Remarque sur un théorème de M. Mullikin, Fund. Math. 6 (1924), 37-38.

5. A. M. Mullikin, Certain theorems relating to plane connected point sets, Trans. Amer. Math. Soc. 24 (1922), 144-162.

6. M. H. A. Newman, Elements of the topologv of plane sets of points, Cambridge Univ. Press, Cambridge, 1961.

Department of Mathematics, Statistics and Computer Science, Marquette University, MiLWAUKEE, WisCONSIN 53233 